\title{
Basal Ganglia Iron in Patients with Multiple Sclerosis Measured with 7T Quantitative Susceptibility Mapping Correlates with Inhibitory Control
}

\author{
(D). Schmalbrock, R.S. Prakash, B. Schirda, A. Janssen, G.K. Yang, M. Russell, M.V. Knopp, A. Boster, J.A. Nicholas, M. Racke,
} and D. Pitt

\begin{abstract}
BACKGROUND AND PURPOSE: T2 hypointensity in the basal ganglia of patients with MS has been associated with clinical progression and cognitive decline. Our objectives were the following: 1) to compare signal in T2WI, R2 (ie, 1/T2), and R2* (ie, 1/T2*) relaxation rates and quantitative susceptibility mapping; and 2) to investigate the associations among MR imaging, clinical scores, and cognitive measures of inhibitory control linked to basal ganglia functioning.
\end{abstract}

MATERIALS AND METHODS: Twenty-nine patients with MS underwent a battery of neuropsychological tests including the Flanker and Stroop tasks. 7T MR imaging included 3D gradient-echo and single-echo multishot spin-echo EPI. Quantitative susceptibility mapping images were calculated by using a Wiener filter deconvolution algorithm. T2WI signal was normalized to CSF. R2 and R2* were calculated by log-linear regression. Average MR imaging metrics for the globus pallidus, putamen, and caudate were computed from manually traced ROls including the largest central part of each structure.

RESULTS: Marked spatial variation was consistently visualized on quantitative susceptibility mapping and T2/T2*WI within each basal ganglia structure. MR imaging metrics correlated with each other for each basal ganglia structure individually. Notably, caudate and putamen quantitative susceptibility mapping metrics were similar, but the putamen R2 was larger than the caudate R2. This finding suggests that tissue features contribute differently to R2 and quantitative susceptibility mapping. Caudate and anterior putamen quantitative susceptibility mapping correlated with the Flanker but not Stroop measures; R2 did not correlate with inhibitory control measures. Putamen quantitative susceptibility mapping and caudate and putamen R2 correlated with the Expanded Disability Status Scale.

CONCLUSIONS: Our study showed that quantitative susceptibility mapping and R2 may be complementary indicators for basal ganglia tissue changes in MS. Our findings are consistent with the hypothesis that decreased performance of basal ganglia-reliant tasks involving inhibitory control is associated with increased quantitative susceptibility mapping.

ABBREVIATIONS: $B$ G = basal ganglia; EDSS = Expanded Disability Status Scale; $G P=$ globus pallidus; $Q S M=$ quantitative susceptibility mapping; $R 2$ = relaxation rate (ie, $1 / \mathrm{T} 2) ; \mathrm{R} 2^{*}=$ relaxation rate $\left(\mathrm{ie}, 1 / \mathrm{T} 2^{*}\right)$

S everal studies have reported changes in $\mathrm{T} 2, \mathrm{~T}^{*}$, and quantitative susceptibility mapping (QSM) in the basal ganglia (BG) of patients with MS compared with healthy controls based on T2 hypointensity. Quantitative CSF-normalized T2 measurements correlated with brain atrophy as measured by volumetric analyses

Received March 5, 2015; accepted after revision July 31.

From the Departments of Radiology (P.S., G.K.Y., M. Russell, M.V.K.), Psychology (R.S.P., B.S., A.J.), and Neurology (A.B., J.A.N., M. Racke), The Ohio State University, Columbus, Ohio; and Department of Neurology (D.P.), Yale School of Medicine, New Haven, Connecticut.

This work was supported by Biogen (US-TSY-10-10047) and the Wright Center of Innovation in Biomedical Imaging at The Ohio State University.

Paper previously presented in part at: European Committee for Treatment and Research in Multiple Sclerosis-Americas Committee for Treatment and Research in Multiple Sclerosis, September, 10-13, 2014; Boston, Massachusetts; and International Society for Magnetic Resonance in Medicine, May 30-June 5, 2015; Toronto, Ontario, Canada.

and with white matter lesion load, ${ }^{1-3}$ and with measures of MS disease burden such as the Expanded Disability Status Scale (EDSS), the timed 25-foot walk test, ${ }^{4,5}$ and cognitive performance. ${ }^{6}$ Change in T2WI hypointensity was reported to be a better predictor of MS disease progression than brain atrophy $y^{5,7}$ and thus may serve as a biomarker for progression and as an outcome measure to assess treatment efficacy. ${ }^{8}$

Although frequently used as a marker for BG iron, T2WI signal is affected not only by iron content but also by a number of other factors, most notably free water mobility. In addition, T2

Please address correspondence to Petra Schmalbrock, PhD, 395 W 12th Ave, 412, Columbus, OH 43210; e-mail: petra.schmalbrock@osumc.edu; @WCIBMI999

- Indicates open access to non-subscribers at www.ajnr.org

三 Indicates article with supplemental on-line tables.

Indicates article with supplemental on-line photos.

http://dx.doi.org/10.3174/ajnr.A4599 
measures may be compromised by fluid motion when the CSF signal is used for normalization. Consequently, a number of other MR imaging methods that are thought to be more sensitive to tissue iron have been pursued, including T2 relaxometry, ${ }^{3,9} \mathrm{~T} 2{ }^{*}$ measurement, ${ }^{10}$ high-pass filtered-phase MR imaging, ${ }^{11}$ and quantitative susceptibility mapping. ${ }^{12}$ These studies and MR imaging-histology comparisons ${ }^{13}$ demonstrated that higher iron content is a prominent factor in BG abnormality in patients with MS.

Furthermore, it has been increasingly recognized that BG structures play a role in both somatomotor and cognitive control processes associated with localized functional topography. ${ }^{14,15}$ Specifically, the BG are implicated in action selection by influencing and modulating activity of the motor cortex and descending motor pathways through inhibitory control. ${ }^{16}$ As such, the abnormal iron increase in the BG of patients with MS may be related to cognitive decline, specifically to deficits in inhibitory control. However, to our knowledge, only 1 study has linked BG iron to cognition in MS. ${ }^{6}$ Additionally, direct comparison between T2 and QSM for measuring BG iron in patients with MS has not yet been published, to our knowledge.

Our study had 2 objectives: 1) to compare T2, T2*, and QSM for assessing BG changes; and 2) to correlate T2, T2*, and QSM to physical and cognitive measures. Specifically, given that the BG play an important role in tasks of inhibitory control, which require suppression of task-irrelevant information to engage correctly in goal-directed, task-relevant behavior, we hypothesized a negative association between iron deposits and neuropsychological measures of inhibitory control.

\section{MATERIALS AND METHODS \\ Patient Selection}

Twenty-nine individuals with relapsing-remitting MS participating in an ongoing longitudinal study were recruited (see On-line Table 1 for demographics and clinical characteristics). All patients signed institutional review board-approved consent forms. EDSS scores were obtained by a neurologist. Thirteen patients were treated with natalizumab; 11 , with glatiramer acetate; 3 , with interferon $\beta$; and 2 were newly diagnosed. On average, patients treated with natalizumab were younger and had shorter MS durations than patients treated with glatiramer acetate. EDSS scores were not significantly different among treatment groups.

\section{MR Imaging Acquisition and Image Processing}

All patients were scanned at 7T (Achieva; Philips Healthcare, Best, the Netherlands) by using a head volume transmit/16-channel receive coil (Nova Medical, Wilmington, Massachusetts). 3Dmultiecho gradient-echo images covering the entire brain were acquired by using TR/flip angle $=24 \mathrm{~ms} / 5^{\circ}, 4$ bipolar gradient echoes with TE $=4-20 \mathrm{~ms}$, acquired voxel size of $0.5 \times 0.5 \mathrm{~mm}$ in-plane and $1-\mathrm{mm}$ section thickness (interpolated to $0.5-\mathrm{mm}$ isotropic), and 9-minute scan time. Complex multicoil data were combined by using scanner software, and real and imaginary data were exported for relaxation rate (ie, $1 / \mathrm{T} 2^{*} ; \mathrm{R} 2^{*}$ ) and QSM processing. T2 data were acquired as 4 separately acquired singleecho multishot spin-echo EPI scans by using $\mathrm{TR}=5000 \mathrm{~ms}$; $\mathrm{TE}=$ $13.5,21,36$, and $50 \mathrm{~ms} ; 1 \times 1 \times 3 \mathrm{~mm}$; and scan times of 1 minute each. Three axial sections through the central part of the BG were acquired. The single-echo sequence was chosen over multiecho to avoid interference from stimulated echoes and because it is more sensitive to diffusion in the inhomogeneous field from tissue microstructure.

Phase maps were calculated from the complex data and unwrapped (FSL PRELUDE, http://www.fmrib.ox.ac.uk/fsl ${ }^{17}$ ). To avoid echo shifts introduced by using a bipolar gradient readout, we used difference images between echo 1 and echo 3 for all further processing. Background field removal was done with a Projection onto Dipole Field. ${ }^{18}$ Image data were masked more tightly than in the published algorithm to handle stronger phase changes near air-tissue interfaces at 7T. Quantitative susceptibility maps were calculated by using Wiener filter deconvolution (Figs 1 and 2). ${ }^{19}$ ROIs in the central part of the right and left globus pallidus (GP), putamen, and caudate were manually traced on 10 sections, including the entire structure seen in the section (Fig 2). ROIs contained 1000-3000 voxels. In addition, the putamen region was split in half, and the anterior and posterior sections were analyzed separately. ROI masks were saved and used to compute average QSM (and SD) and T2*WI signal at the 4 different TEs. Mean $\mathrm{T}^{*}$ and SDs were computed from the average signals with log-linear regression.

For T2 analysis, ROIs were manually traced on the $\mathrm{TE}=36 \mathrm{~ms}$ images. These ROIs were used to calculate signal for all TEs. Quantitative T2 mean and SDs were computed by log-linear regression. To compare quantitative relaxometry with CSF-normalized T2-weighting, ${ }^{1,2,4,6}$ we also traced ROIs in the anterior horns of the ventricles on the $\mathrm{TE}=36 \mathrm{~ms}$ images and computed the ratio of $\mathrm{BG}$ over CSF signal (ratio $=\mathrm{S}_{\mathrm{BG}} / \mathrm{S}_{\mathrm{CSF}}$ ) and its $\mathrm{SD}$.

\section{Neuropsychological Testing}

All participants completed neuropsychological assessments designed to measure general cognitive functioning and specific measures that assess functioning on tasks that are subserved by the BG. To examine general cognitive decline, primarily relevant for the ongoing longitudinal study, we administered the Mini-Mental State Examination, the Wechsler Test of Adult Reading, and the Brief Repeatable Battery (On-line Table 2), ${ }^{20,21}$ establishing a cognitive baseline for each participant. Participants additionally underwent testing with computerized versions of Flanker and Stroop tasks to assess inhibitory control (On-line Table 3). ${ }^{21}$

\section{Statistical Analysis}

Statistical analyses were performed with Matlab (MathWorks, Natick, Massachusetts). All demographic, MR imaging, and neuropsychological data were tested for outliers, defined as any $z$ score deviation of $>2.5$ SDs from the mean. Normality was checked by using the Shapiro-Wilk test. In our data, disease duration and Flanker interference were found to be positively skewed and were corrected with a square root transformation. EDSS data on 1 participant were missing, and we estimated the EDSS of this participant by using the fitted linear regression between disease duration and EDSS. Data from 2 participants were excluded from the neuropsychological analyses, 1 for the Flanker task and 1 for the Stroop task, due to patients performing below chance on all conditions of the task. 


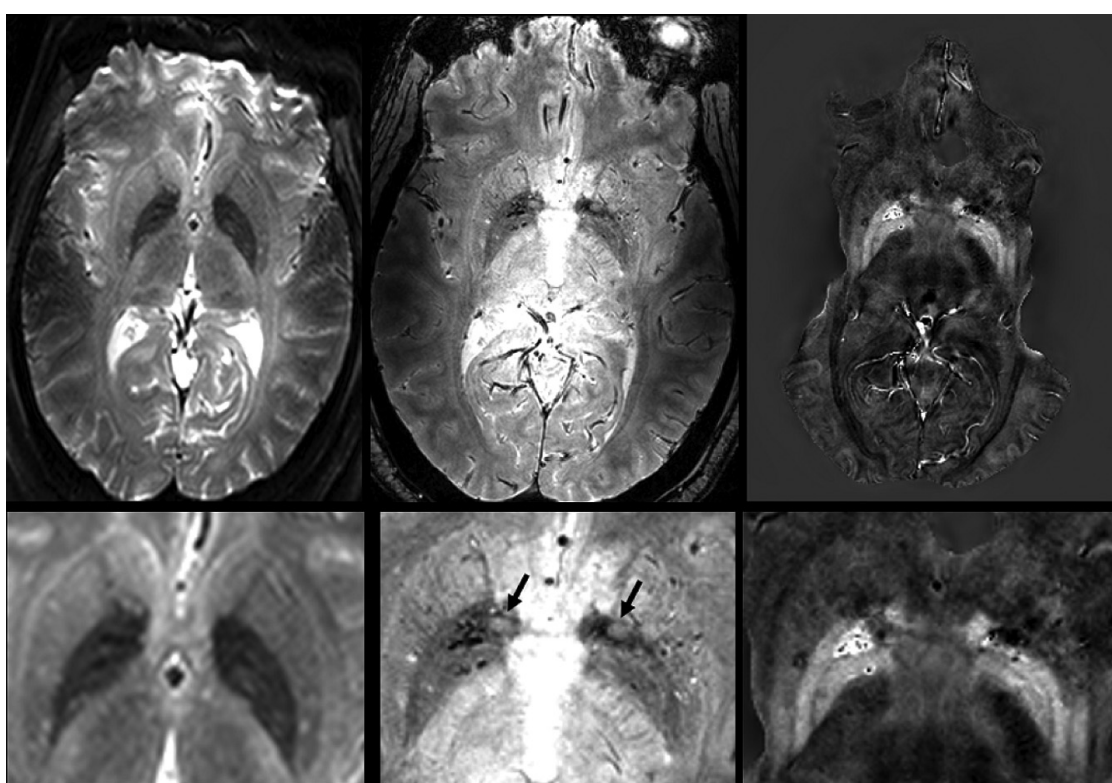

FIG 1. T2-weighted (TE $=36 \mathrm{~ms}), T 2{ }^{*}$-weighted ( $\mathrm{TE}=14 \mathrm{~ms}$ ), and QSM images (left, middle, right) of a 44-year-old female patient with MS (EDSS = 3; duration, 1.7 years). The T2WI multishot spin-echo EPI and T2*WI have some distortion due to susceptibility near air spaces in the sinus. A significant part of the temporal lobes is masked in QSM to avoid processing artifacts, but the BG are not obscured. Magnified T2/T2*-weighted and QSM images show many subtle structures. The internal and external GPs are clearly separated, especially on QSM. In this younger patient, putamen iron is higher only in posterolateral regions. Veins are dark on T2*WI and bright on QSM. Several high-iron regions (dark on T2WI and T2*WI, bright on QSM) are seen in the globus pallidus. Bright spots in the putamen and GP are dark on QSM and may be arterial inflow or small high T2 lesions (mobile water). Two areas at the anterior GP edge (arrows, gray on T2*WI, dark on QSM) are WM regions of the anterior commissure; they are not seen on T2WI due to the larger section thickness.

Pearson correlations were computed between all measured variables. QSM, R2 (ie, 1/T2), and R2* (ie, 1/T2*) relaxation rates increased with age, and the EDSS score increased with disease duration. To remove these dependencies, we subtracted a linear regression fit of QSM, R2/R2* with age, and EDSS with duration from the measured data. Semipartial correlations were conducted between cognitive (Flanker), duration-adjusted physical (EDSS), and age-adjusted MR imaging measures.

\section{RESULTS}

\section{Visual Assessment of the MR Imaging Data}

Image Quality. QSM processing produced high-quality images rich in anatomic details with minimal blurring and artifacts (Figs 1 and 2 and On-line Fig 1). Some streaking from QSM processing was especially visible in coronal reformatted images (On-line Fig 1). Because we chose a very conservative threshold for the background field removal algorithm, ${ }^{18}$ significant parts of temporal lobes and hippocampal regions were masked, but the BG were retained. Motion artifacts were observed in some patients but did not preclude quantitative analysis. Multishot T2-weighted EPI had significant distortion near air/tissue interfaces (Fig 1). This occasionally led to banding artifacts extending into the brain, but they did not hinder quantitative $\mathrm{T} 2$ analysis.

Image Characteristics. QSM and signal on T2/T2*WI were not homogeneous throughout the GP, putamen, and caudate. Internal and external GPs could be distinguished on original axial and reformatted coronal images in most patients (Figs 1 and 2 and
On-line Fig 1). In the GP, bright "spots" were frequently seen on QSM with values 1-3 SDs higher than those of surrounding tissue. These regions were dark on T2/T2*WI (Figs 1 and 2 and On-line Fig 1). Sometimes, it was possible to follow these bright-QSM/dark-T2 features through adjacent sections, thus identifying them as vasculature, but often they appeared isolated. Compared with superior GP regions, inferior regions were darker on $\mathrm{T} 2 / \mathrm{T} 2{ }^{*} \mathrm{WI}$ and brighter on QSM (Fig 2 and On-line Fig 1). In the putamen, bright or dark spots were also observed, but most notably, there was a gradual change in signal from darker posterolateral to brighter anteromedial regions on $\mathrm{T} 2 / \mathrm{T} 2{ }^{*} \mathrm{WI}$, with an analogous pattern from bright to dark in QSM. In the caudate, the posteromedial tips were dark on T2/ $\mathrm{T} 2{ }^{*} \mathrm{WI}$ and bright on QSM and seemed to connect to fine vessels visibly extending into the caudate.

These spatial patterns were seen with all imaging methods, T2/T2*WI and QSM, and remained stable with time (On-line Fig 1), ascertaining that regional variations within each BG structure reflected true tissue differences and were not merely due to SNR limitations, artifacts, or processing errors.

\section{Comparison of Quantitative Measures for QSM, T2, and $\mathrm{T}^{*}$ *}

Measurement Precision. To assess measurement variability, we calculated the average coefficient of variation over all patients and all structures. The coefficient of variation was highest for CSF-normalized T2WI (20\%) and lowest for T2 and T2* relaxometry (both $3 \%$ ). At $11.5 \%$, the coefficient of variation for QSM was fairly high because local differences were amplified in QSM.

Relations among MR Imaging Measures. Table 1 shows mean QSM, R2* and R2. Note that the putamen and caudate QSM were similar, whereas caudate R2 was lower than the putamen R2. Table 2 lists correlation coefficients between MR imaging measures; all were high ( $P$ values $<10^{-3}$, data in On-line Fig 2$)$. Most interesting, correlation coefficients between R2 and QSM were lowest, and linear regression slopes were significantly different among GP structures (On-line Fig 2). Also note that correlation coefficients and linear regression slopes were lower for the caudate than the GP and putamen. Last, high correlation between CSF-normalized T2WI and quantitative T2 indicated that simple normalization can be a valid measure, albeit with higher measurement error.

Table 2 also lists fit coefficients for multivariable linear regres-

AJNR Am J Neuroradiol 37:439-46 Mar 2016 www.ajnr.org

441 

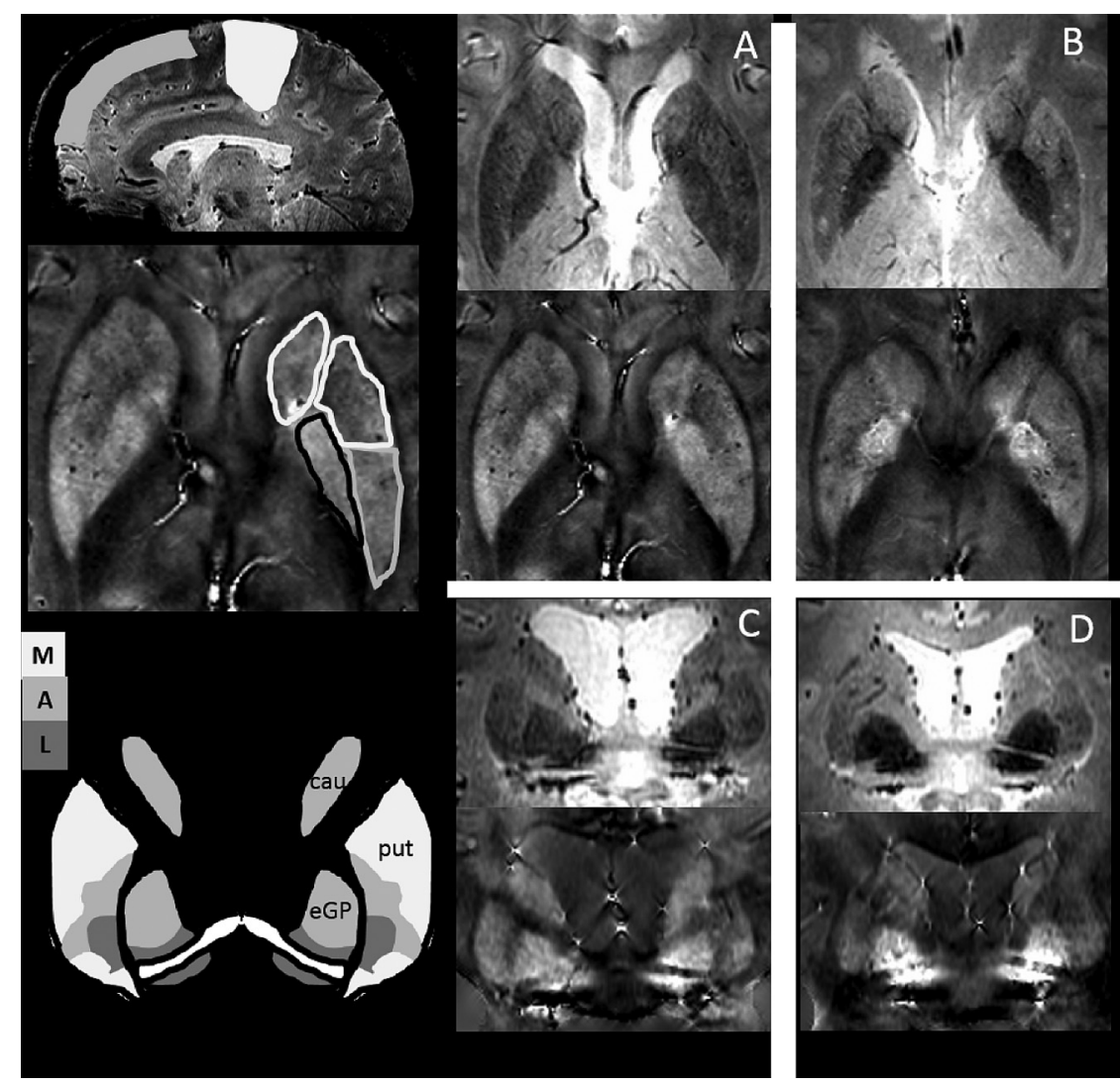

FIG 2. Schematic representation of published basal ganglia-cortical connections $s^{14,15}$ and sample T2*-weighted and QSM images for 4 different patients. On the left side is a schematic representation of frontal and sensorimotor areas indicated on a sagittal view (top); sample ROIs used for the analysis in this study are shown on an axial view (middle). As indicated, the putamen ROI was split in half into anterior and posterior sections, which were separately analyzed. At the left bottom is a schematic representation of BG functional areas sketched from published calbindin stains. ${ }^{14}$ Our GP and putamen ROls were typically selected on more inferior axial sections than the caudate ROls so that GP and putamen areas included mixed functional areas, whereas our caudate ROI was predominantly in a cognitive area. The schematic depictions are contrasted with data from 4 different patients on the right. Patient A (a 33-year-old woman; EDSS = 4; Flanker, 37 $\mathrm{ms}$ ) has lower physical but slightly higher cognitive performance than patient B (a 30-year-old woman; EDSS = 2; Flanker, $57 \mathrm{~ms}$ ). QSM is higher in the posterior putamen for patient A. Coronal reformatted images of patient C (a 50-year-old woman; EDSS = 5; Flanker, $22 \mathrm{~ms}$ ) who has worse motor but better cognitive function than patient $\mathrm{D}$ (a 50-year-old woman; EDSS = 0; Flanker, 108 $\mathrm{ms}$ ) show higher QSM in the putamen, but lower QSM in the external GP for patient C compared with patient D. Again, there is some spatial correspondence between high QSM and functional deficits in these patients.

Table 1: Mean QSM, R2, and R2* for BG substructures across patients

\begin{tabular}{lccccc}
\hline & GP & Putamen & Caudate & Aput & \multicolumn{1}{c}{ Pput } \\
\hline QSM & $0.159 \pm 0.031$ & $0.064 \pm 0.023$ & $0.068 \pm 0019$ & $0.050 \pm 0.019$ & $0.095 \pm 0.038$ \\
R2 $^{*}$ & $0.096 \pm 0.013$ & $0.061 \pm 0.011$ & $0.051 \pm 0.009$ & $0.059 \pm 0.010$ & $0.066 \pm 0.016$ \\
R2 & $0.049 \pm 0.004$ & $0.036 \pm 0.004$ & $0.029 \pm 0.002$ & $0.034 \pm 0.004$ & $0.039 \pm 0.0050$
\end{tabular}

Note:-Aput indicates cognitive anterior part of putamen, region predominantly connected to frontal cortex; Pput, motor posterior part of putamen, region predominantly connected to sensorimotor cortex.

sion of R2* with both QSM and R2 (by using intercept zero). These coefficients were used to estimate the relative contribution from R2 and QSM to R2* (Table 2). Remarkably, QSM contributed most prominently to $\mathrm{R}^{*}$ in the GP and (motor cortex-connected) posterior putamen, whereas $\mathrm{R} 2$ dominated in the caudate and (cognitive cortex-connected) anterior putamen. Taken together, the observed relations between the different MR imaging measures indicated that QSM and R2 carried somewhat different information about tissue microstructure, whereas $\mathrm{R} 2{ }^{\star}$ was a mixed measure.
Associations among Demographic, Clinical, and Neuropsychological Parameters. Only disease duration and EDSS were strongly correlated; neither Flanker nor Stroop measures correlated with age, disease duration, or EDSS (Table 3). Notably, in our cohort of patients with MS, Flanker and Stroop measures did not correlate.

Associations among MR Imaging Metrics and Demographic, Clinical, and Inhibitory Control Measures. Significant correlations with age and QSM were observed for all regions and for R2 for the GP and putamen but not the caudate (Table 4). Associations were weaker for R2*. Age-related trends followed published data for healthy subjects in the age range of $30-60$ years. $^{22}$ Compared with published data, ${ }^{22}$ GP and caudate QSM were higher in patients with MS than in controls (Online Fig 3). 7T data for R2 are not available, but age-related trends have been shown at a lower field strength. Caudate QSM also correlated with disease duration (Table 4).

EDSS correlated significantly with R2 in the caudate and putamen, but not the GP (Table 4). Most interesting, no bivariate correlations between EDSS and QSM or R2* were found without controlling for covariates. After we corrected for age in MR imaging measures and duration in EDSS, stronger correlations were revealed (Table 5). There was a significant correlation between EDSS and R2 for the caudate and putamen (with both putamen subregions). Additional associations between EDSS and QSM ensued and were most prominent for the posterior putamen connected to the motor cortex (Table 5). Still, no correlations were found between EDSS and QSM in the anterior (cognitive) putamen and caudate (On-line Fig 4).

Regarding associations between measures of inhibitory control and MR imaging, bivariate analyses without correction for covariates showed only an association between the Flanker measure and QSM in the caudate and anterior putamen (Table 4). We did not find any associations between QSM and the Stroop task. R2 and R2* did not correlate with Flanker or Stroop measures for any BG structure. After we corrected QSM and R2 for age, correlations between the Flanker measure and the caudate and anterior putamen QSM became stronger, but still there was no association with R2 (Table 5 and On-line Fig 4). 
Table 2: Bivariate Pearson correlation coefficients between MRI measures and multivariable linear regression of R2* with QSM and R2 using zero intercept (R2* $=$ a1QSM + a2R2)

\begin{tabular}{|c|c|c|c|c|c|}
\hline & GP & Putamen & Caudate & Aput & Pput \\
\hline \multicolumn{6}{|l|}{ MRI } \\
\hline R2 (QSM) & $0.51^{\mathrm{a}}$ & $0.72^{b}$ & $0.52^{\mathrm{a}}$ & $0.67^{\mathrm{a}}$ & $0.72^{\mathrm{a}}$ \\
\hline $\mathrm{R}^{*}$ (QSM) & $0.73^{\mathrm{b}}$ & $0.81^{b}$ & $0.47^{\mathrm{a}}$ & $0.71^{\mathrm{a}}$ & $0.83^{\mathrm{a}}$ \\
\hline$R 2 *(R 2)$ & $0.55^{\mathrm{b}}$ & $0.78^{\mathrm{b}}$ & $0.48^{\mathrm{a}}$ & $0.75^{\mathrm{a}}$ & $0.75^{\mathrm{a}}$ \\
\hline CSF-T2WI (T2) & $0.71^{\mathrm{b}}$ & $0.85^{b}$ & $0.59^{b}$ & $0.79^{\mathrm{b}}$ & $0.87^{b}$ \\
\hline \multicolumn{6}{|c|}{ Multivariable linear regression for $\mathrm{R} 2$ * } \\
\hline$a_{1}$ & $0.28 \pm 0.04^{b}$ & $0.22 \pm 0.04^{b}$ & $0.15 \pm 0.06^{c}$ & $0.21 \pm 0.05^{\mathrm{a}}$ & $0.25 \pm 0.04^{b}$ \\
\hline$a_{2}$ & $1.05 \pm 0.14^{\mathrm{b}}$ & $1.31 \pm 0.07^{b}$ & $1.42 \pm 0.16^{\mathrm{b}}$ & $1.42 \pm 0.08^{b}$ & $1.08 \pm 0.10^{\mathrm{b}}$ \\
\hline Fractional & QSM: 47\% & QSM: 23\% & QSM: $20.0 \%$ & QSM: 18\% & QSM: $36 \%$ \\
\hline Contributions & R2: $53 \%$ & R2: $77 \%$ & R2: $80.0 \%$ & R2: $82 \%$ & R2: 64\% \\
\hline
\end{tabular}

Note:-Aput indicates cognitive anterior part of putamen, region predominantly connected to frontal cortex; Pput, motor posterior part of putamen, region predominantly connected to sensorimotor cortex.

${ }^{\text {a }} P<10^{-3}$.

${ }^{\mathrm{b}} P<10^{-6}$.

${ }^{c} P=.02$.

Table 3: Pearson correlation coefficients ( $P$ values) for demographic and neuropsychological measures

\begin{tabular}{|c|c|c|c|c|c|}
\hline & Age & Duration & EDSS & Flanker & Stroop \\
\hline Age & 1 & & & & \\
\hline Duration & $0.36(.06)$ & 1 & & & \\
\hline $\mathrm{EDSS}^{\mathrm{a}}$ & $0.30(.11)$ & $0.61(.0005)^{d}$ & 1 & & \\
\hline Flanker ${ }^{\mathrm{b}}$ & $0.01(.95)$ & $0.26(.18)$ & $0.23(.23)$ & 1 & \\
\hline Stroop ${ }^{c}$ & $0.07(.74)$ & $-0.08(.68)$ & $-0.02(.94)$ & $0.03(.90)$ & 1 \\
\hline
\end{tabular}

${ }^{a}$ Using estimates for missing EDSS scores.

b For the Flanker task, 1 participant performed below chance $(n=28)$.

${ }^{\mathrm{c}}$ For the Stroop task, data for 1 participant were lost, and 1 performed below chance $(n=27)$.

${ }^{\mathrm{d}}$ Statistically significant at $P<.05$.

Table 4: Bivariate Pearson correlation coefficients ( $P$ values) ( $n=29$ except as noted)

\begin{tabular}{|c|c|c|c|c|c|}
\hline & Age & Duration $^{\mathrm{a}}$ & EDSS & Flanker $^{b}$ & Stroop ${ }^{c}$ \\
\hline \multicolumn{6}{|l|}{ QSM } \\
\hline GP & $0.36(.059)$ & $0.10(.60)$ & $0.06(0.75)$ & $0.25(.20)$ & $-0.18(.36)$ \\
\hline Put & $0.57(.001)^{f}$ & $0.04(.85)$ & $0.31(0.09)$ & $0.3(.12)$ & $0.01(.97)$ \\
\hline Cau & $0.41(.02)^{f}$ & $0.42(.02)^{f}$ & $0.24(0.20)$ & $0.47(.01)^{f}$ & $0.13(.50)$ \\
\hline Aput & $0.55(.0015)^{f}$ & $0.05(.81)$ & $0.30(0.11)$ & $0.38(.045)^{f}$ & $0.00(.99)$ \\
\hline Pput & $0.57(.001)^{f}$ & $0.09(.65)$ & $0.35(0.06)$ & $0.23(.25)$ & $-0.01(.94)$ \\
\hline \multicolumn{6}{|l|}{ R2 } \\
\hline$G^{d}$ & $0.32(.12)$ & $0.08(.68)$ & $0.18(0.38)$ & $0.32(.11)$ & $0.065(.76)$ \\
\hline Put $^{e}$ & $0.47(.01)^{f}$ & $0.09(.65)$ & $0.43(0.02)^{f}$ & 0.31 (.11) & $0.21(.34)$ \\
\hline $\mathrm{Cau}^{\mathrm{e}}$ & $0.37(.06)$ & $0.29(.15)^{\prime}$ & $0.58(.001)^{f}$ & 0.31 (.11) & $0.07(.75)$ \\
\hline Aput $^{\mathrm{e}}$ & $0.44(.02)^{f}$ & $0.12(.54)$ & $0.48(.01)^{f}$ & $0.35(.08)$ & $0.17(.44)$ \\
\hline Pput $^{\mathrm{e}}$ & $0.47(.01)^{f}$ & $0.01(.96)$ & $0.32(.04)^{f}$ & $0.21(.29)$ & $0.28(.18)$ \\
\hline \multicolumn{6}{|l|}{$\mathrm{R}^{*}{ }^{*}$} \\
\hline GP & $0.14(.45)$ & $-0.23(.22)$ & $-0.20(.29)$ & $0.2(.3)$ & $-0.24(.22)$ \\
\hline Put & $0.38(.047)^{f}$ & $-0.12(.51)$ & $0.25(.19)$ & $0.34(.075)$ & $-0.02(.92)$ \\
\hline Cau & 0.29 (.12) & $-0.02(.90)$ & $0.24(.20)$ & $0.29(.13)$ & $0.27(.17)$ \\
\hline Aput & 0.30 (.11) & $-0.15(.43)$ & $0.26(.16)$ & $0.37(.055)$ & $-0.04(.84)$ \\
\hline Pput & $0.45(.01)^{f}$ & $-0.06(.74)$ & $0.17(.36)$ & $0.23(.23)^{\prime}$ & $-0.00(.99)$ \\
\hline
\end{tabular}

Note:-Cau indicates caudate; Put, putamen; Aput, cognitive anterior part of putamen, region predominantly connected to frontal cortex; Pput, motor posterior part of putamen, region predominantly connected to sensorimotor cortex.

a Using square root transformation for non-normal duration data.

b Using square root transformation for non-normal Flanker measure $(n=28)$.

c Stroop measure $(n=27)$.

${ }^{\mathrm{d}}$ For GP R2 $n=27$ so that R2-GP versus Flanker $(n=26)$.

${ }^{e}$ For putamen and caudate R2 $n=28$ so that R2-Put, R2-Cau versus Flanker ( $\left.n=27\right)$.

f Statistically significant at $P<.05$.

In summary, the cognitive measure, Flanker interference, showed a stronger association with QSM in the caudate and anterior putamen, whereas the physical measure, EDSS, showed a stronger association with R2 but not R2*.

\section{DISCUSSION}

Our study had 2 goals: first, to compare QSM, T2*, and T2 for assessing BG changes in patients with MS and second to study associations among MR imaging metrics, clinical scores, and cognitive measures of inhibitory control.

\section{Comparison of MR Imaging Measures}

Our study found visual correspondence (ie, similar spatial patterns within each BG region) among QSM, T2*WI, and T2WI. MR imaging measures increased with age, and the correlation among the MR imaging metrics was high. Notably, the dynamic range of QSM was twice as high as that of R2 and R2*.

However, there were several prominent differences. The caudate QSM was higher than the putamen QSM, whereas the caudate R2 was lower than putamen R2. Postmortem studies of tissue iron showed the highest iron content in the GP, followed by the putamen, and the lowest iron content in the caudate. ${ }^{13}$ This order was maintained in QSM studies of healthy subjects. ${ }^{12,23}$ Similar to our study findings, Rudko et $\mathrm{al}^{23}$ demonstrated higher caudate QSM in patients with MS, though overall, their QSM values were lower in their younger subject population. Conversely, the study of Langkammer et $\mathrm{al}^{12}$ did not show the relative increase in caudate QSM in patients with MS. Furthermore, we observed almost a 2-fold higher QSM in the posterior compared with the anterior putamen, whereas $\mathrm{R} 2$ was only 15\% higher (Table 1).

An increase in QSM may be due to higher iron or lower myelin content, whereas larger $\mathrm{R} 2$ and $\mathrm{R} 2{ }^{\star}$ are due to higher iron and higher myelin. Thus our finding of relatively higher QSM in the caudate is consistent with increased iron content and/or decreased myelin, both suggesting greater tissue damage in the caudate. Increased QSM in the posterior compared with the anterior putamen suggests higher iron content in the posterior putamen.

Multivariate linear regression of R2* with QSM and R2 (Table 2 ) suggests that $R 2^{\star}$ is a mixed measure. Even though our simple linear model does not reflect the complexities of the true relationships among R2, R2*, and QSM, our analysis, nevertheless, demonstrates that different BG structures have very different relative contributions from QSM and R2. It also suggests that R2* is a less sensitive measure than QSM, whereas R2 may provide complementary information. Thus, our finding that CSF-normalized T2WI correlates well with T2-relaxometry is of interest because it indicates that fast and simple T2WI can serve as a substitute for time-consuming $\mathrm{T} 2$ relaxometry. 
Table 5: Correlation coefficients ( $P$ values) for semipartial correlations after adjusting MRI parameters for age and EDSS for disease duration

\begin{tabular}{|c|c|c|c|c|c|}
\hline & GP & Put & Cau & Aput & Pput \\
\hline Sqrt (Flanker) vs age-adjusted QSM & $0.26(.18)$ & $0.36(.063)$ & $0.51(.0058)^{a}$ & $0.45(.016)^{\mathrm{a}}$ & $0.26(.17)$ \\
\hline Sqrt (Flanker) vs age-adjusted R2 & $0.33(.09)$ & $0.34(.08)$ & $0.31(.11)$ & $0.37(.056)$ & $0.23(.25)$ \\
\hline Duration-adjusted EDSS vs age-adjusted QSM & $0.00(.98)$ & $0.39(.036)^{\mathrm{a}}$ & $0.01(.95)$ & $0.34(.07)$ & $0.40(.032)^{\mathrm{a}}$ \\
\hline Duration-adjusted EDSS vs age-adjusted R2 & $0.16(.43)$ & $0.51(.0059)^{\mathrm{a}}$ & $0.46(.014)^{\mathrm{a}}$ & $0.51(.0054)^{\mathrm{a}}$ & $0.44(.019)^{\mathrm{a}}$ \\
\hline
\end{tabular}

Note:-Sqrt indicates the square root transformation used for the non-normal distributed Flanker test metrics; Cau, caudate; Put, putamen; Aput, cognitive anterior part of putamen, region predominantly connected to frontal cortex; Pput, motor posterior part of putamen, region predominantly connected to sensorimotor cortex.

${ }^{\text {a }}$ Statistically significant at $P<.05$.

In summary, both the larger dynamic range and the small fractional contribution of QSM to R2* suggest that QSM is a more sensitive and perhaps more specific measure than R2*. Future studies modeling R2, R2*, and QSM based on tissue microstructure, including paramagnetic iron predominantly located in oligodendrocytes and diamagnetic myelin, may further elucidate the relative importance of QSM versus R2.

\section{Association of MR Imaging with Clinical and Cognitive Measures}

An interesting finding of our study was that cognitive performance measured by the Flanker test was associated with QSM but not R2 in the caudate and anterior putamen, whereas EDSS was preferentially associated with R2.

Association of EDSS with MR Imaging Metrics. EDSS is a measure of physical disability in MS. In our population, the EDSS score was strongly correlated with disease duration. We found that EDSS was associated with R2 for the caudate and putamen, with QSM in the posterior putamen, but not the globus pallidus. Conversely, Rudko et $\mathrm{al}^{23}$ found an association between EDSS for both QSM and R2* in all BG regions. This discrepancy may be due to differences in population characteristics; the patients of Rudko et al were younger and had shorter disease duration; these features were not associated with EDSS. Additionally, their average ROI analysis included the full BG structures as defined by atlas data, whereas our study evaluated the central part seen on axial images. Thus, our MR imaging measures may have included specific functionally connected BG regions, whereas the data of Rudko et al included a mixture of all functional BG regions.

Association of the Flanker Task with MR Imaging. We found a positive association between the Flanker task performance and QSM for the caudate and anterior putamen, but not for the posterior putamen and GP. No associations were found with R2.

These findings are consistent with preferential connectivity patterns linking the caudate and anterior putamen to frontal brain areas necessary for inhibitory control processing (Fig 2). ${ }^{14,15}$ In contrast, the posterior putamen is connected to the motor-sensory cortex, and the GP is linked to motor, cognitive, and limbic areas. ${ }^{14,15}$ In addition, comparison of MR imaging metrics suggests that QSM is perhaps most sensitive for the detection of tissue damage, especially in the caudate. To the best of our knowledge, only 1 prior study has examined associations between T2 hypointensity and cognitive performance, finding an association between a composite neuropsychological score and CSF-normalized T2WI in the GP. ${ }^{6}$ Differences in the neuropsychological battery may have contributed to the observed discrepancy.

Future studies are needed to confirm our initial results. First, our group of patients with MS $(n=29)$ is too small to firmly establish the validity of associations observed in our study. Studies with a larger MS population and/or longitudinal studies are needed. Furthermore, our study did not include healthy subjects, though our MR imaging metrics followed previously published trends. ${ }^{22-26}$ However, the notion that changes in QSM and R2 in $\mathrm{BG}$ areas preferentially connected to cognitive cortical areas, which may relate to cognitive performance, needs to be further established in healthy subjects.

Flanker versus Stroop Tasks. We expected to find associations between Flanker and Stroop task measures and MR imaging metrics. In contrast, our results did not provide evidence for an association between Flanker and Stroop measures or for the Stroop and any MR imaging measure. Although both the Flanker and Stroop tasks are subsumed under the umbrella term of "inhibitory control," these 2 widely used measures tap into interrelated facets of interference suppression and response inhibition, respectively. "Interference suppression" refers to the ability to suppress task-irrelevant information in favor of task-relevant information, whereas "response inhibition" taps into the ability to suppress a dominant response in favor of a nondominant response. ${ }^{27,28}$ Although both of these facets of inhibitory control rely on the frontal-striatal circuitry ${ }^{29}$ and the BG have been implicated in behavioral performance on these measures, we did not find an association between MR imaging metrics in BG and Stroop performance. This dissociation suggests that there may be some specificity to BG functioning in individuals with MS, and future research could more clearly parse the involvement of the BG substructures in different facets of inhibitory control for this population.

\section{Regional Variability}

Another prominent feature in our high-resolution 7T data was the pronounced spatial variability observed in QSM and T2/ $\mathrm{T} 2{ }^{\star} \mathrm{WI}$, specifically the posterolateral-to-anteromedial signal change in the putamen and focal signal differences most often seen in the GP. Earlier studies by using low-resolution T2WI did not report such findings. ${ }^{1,2,5,6}$ However, several more recent studies described analogous spatial variability in healthy aging ${ }^{22,24,26}$ and related it to cognitive decline in aging. ${ }^{25}$ A study by Zivadinov et $\mathrm{al},{ }^{11}$ using analysis methods emphasizing focal high iron over global/average iron in the BG, found a strong differentiation between healthy controls and patients with MS. Rudko at al ${ }^{23}$ also found similar patterns in voxelwise $z$ score maps comparing healthy controls and patients with MS. Mechanisms for variable iron deposition are not known, but it was suggested that they coincide with lenticulostriate arterioles penetrating the $\mathrm{BG},{ }^{26,30}$ 
indicative of mineralization, including iron encrustation and/or calcification near vessels.

In future studies, it will be instructive to examine how these observed regional MR imaging changes relate to BG functional connections and clinical and cognitive decline in MS. For example, the cases in Fig 2 may suggest that it is feasible to find a visual scoring system for characterizing signal change in BG subregions defined by prior knowledge of BG functional subdivisions. ${ }^{14,15}$ Better yet, subject-specific BG-cortex connections could be identified by simultaneous DTI studies and used to define specific ROIs for QSM and R2 tissue characterization. Finally, voxelwise correlation of MR imaging with clinical and cognitive measures could be used in future studies to better establish BG functionality as a biomarker of disease progression.

We expect that future work by using data analyses that takes into account both local patterns of iron increase and BG functional topography will be the key to gaining additional insight into the role of BG iron in MS-related clinical and cognitive decline and disease progression. High-resolution 7T is uniquely suited for this purpose.

\section{CONCLUSIONS}

Our study showed that both QSM and R2 are indicators for tissue changes in the BG of patients with MS and may provide somewhat complementary information. QSM is more sensitive than R2*, which is a composite measure of R2 and QSM. We substantiated our hypothesis that QSM-based BG assessment can reveal tissue changes that are implicated in BG-reliant tasks of inhibitory control. If the results from our initial cross-sectional study are replicated in future research with larger populations and/or are extended through longitudinal studies, QSM-based MR imaging can be established as a sensitive biomarker for cognitive decline in MS and serve as an outcome measure to determine treatment efficacy.

Disclosures: Petra Schmalbrock—RELATED: Grant: Biogen Idec*; Support for Travel to Meetings for the Study or Other Purposes: Biogen Idec.* Ruchika S. PrakashRELATED: Grant: Biogen Idec (partial funding); Support for Travel to Meetings for the Study or Other Purposes: Biogen Idec (partial funding). Brittney Schirda—RELATED: Grant: Biogen Idec*; Support for Travel to Meetings for the Study or Other Purposes: Biogen Idec (partial funding).* Alisha Janssen—RELATED: Grant: Biogen Idec*; Fees for Participation in Review Activities such as Data Monitoring Boards, Statistical Analysis, Endpoint Committees, and the Like: Biogen Idec.* Grant K. Yang-RELATED: Grant: Goldwater Scholarship Program. Mary RussellRELATED: Grant: Biogen Idec*; Support for Travel to Meetings for the Study or Other Purposes: Biogen Idec grant.* Michael V. Knopp—RELATED: Grant: Ohio Third Frontier TECH 11-044. * Aaron Boster-UNRELATED: Consultancy/Honoraria: Teva, Biogen, Novartis, Genzyme, Mallinckrodt, Medtronic. Jacqueline A. Nicholas—UNRELATED: Consultancy: Novartis, Teva, Genzyme, Biogen Idec; Grants/Grants Pending: National Multiple Sclerosis Society*; Payment for Lectures (including service on Speakers Bureaus): Novartis, Teva, Biogen Idec, Genzyme. Michael Racke-UNRELATED: Board Membership: Editorial Boards: Annals of Neurology, JAMA Neurology, Annals of Clinical and Translational Neurology, Journal of Neuroimmunology (editor-in-chief), Therapeutic Advances in Neurologic Disorders; Other: 2013-15 Research Program Advisory Committee of the National Multiple Sclerosis Society, 2015 Americas Committee for Treatment and Research in Multiple Sclerosis Advisory Committee, International Society of Neuroimmunology Advisory Board, Consortium of Multiple Sclerosis Centers (Treasurer/Executive Committee, 2010-present), MS Section of the American Academy of Neurology (Executive Committee, 2014-15), Comments: Other boards unpaid but travel/lodging often supported to attend meetings; Grants/Grants Pending: Novartis, ${ }^{*}$ Genentech, ${ }^{*}$ DioGenix, ${ }^{*}$ Comments: Money paid to Ohio State for participation in clinical trials; Consulting Fee or Honorarium: Novartis, Genentech, Biogen Idec, Teva Neuroscience, Comments: Honoraria received for consulting; Other: Journal of Neuroimmunology, JAMA Neurol- ogy, Comments: Money received for serving as editor-in-chief (UNI) or editorial board member (IAMA Neuro); Payment for Lectures (including service on Speakers Bureaus): Honoraria, travel, and/or lodging from multiple institutions for giving lectures (University of Texas-Houston, CME Outfitters, National Institutes of Health, American Academy of Neurology, Allegheny General Hospital, Consortium of Multiple Sclerosis Centers, European Academy of Neurology, Lingnan International Neurological Summit Forum, Hunan Central University, Peking Union Medical College). David Pitt-UNRELATED: Payment for Lectures (including service on Speakers Bureaus): Biogen Idec. *Money paid to the institution.

\section{REFERENCES}

1. Bakshi R, Benedict RHB, Bermel RA, et al. T2 hypointensity in the deep gray matter of patients with multiple sclerosis: a quantitative magnetic resonance imaging study. Arch Neurol 2002;59:62-68 CrossRef Medline

2. Bermel RA, Puli SR, Rudick AA, et al. Prediction of longitudinal brain atrophy in multiple sclerosis by gray matter magnetic resonance imaging T2 hypointensity. Arch Neurol 2005;62:1371-76 CrossRef Medline

3. Zhou FQ, Zee CS, Gong HH, et al. Differential changes in deep and cortical gray matters in patients with MS: a quantitative magnetic resonance imaging study. J Comput Assist Tomog 2010;34:413-36 CrossRef Medline

4. Toja CW, Benedict RH, Weinstock-Guttmann B, et al. MRI T2 hypointensity of the dentate nucleus is related to ambulatory impairment in multiple sclerosis. J Neurol Sci 2005;234:17-24 CrossRef Medline

5. Zhang Y, Metz LM, Yong VW, et al. 3T deep gray matter T2 hypointensity correlates with disability over time in stable relapsing-remitting multiple sclerosis: a 3-year pilot study. J Neurol Sci 2010; 297:76-81 CrossRef Medline

6. Brass SD, Benedict RH, Weinstock-Guttman B, et al. Cognitive impairment is associated with subcortical magnetic resonance imaging grey matter T2 hypointensity in multiple sclerosis. Mult Scler 2006;12:437-44 CrossRef Medline

7. Neema M, Arora A, Healy BC, et al. Deep gray matter involvement on brain MRI scans is associated with clinical progression in multiple sclerosis. J Neuroimaging 2009;19:3-8 CrossRef Medline

8. Pawate S, Wang L, Song Y, et al. Analysis of T2 intensity by magnetic resonance imaging of deep gray matter nuclei in multiple sclerosis patients: effect of immunomodulatory therapies. J Neuroimaging 2012;22:137-44 CrossRef Medline

9. Burgetova A, Seidl Z, Krasensky J, et al. Multiple sclerosis and the accumulation of iron in the basal ganglia: quantitative assessment of brain iron using MRI t(2) relaxometry. Eur Neurol 2010;63: 136-43 CrossRef Medline

10. Walsh AL, Blevins G, Lebel RM, et al. Longitudinal MR imaging of iron in multiple sclerosis: an imaging marker of disease. Radiology 2014;270:186-96 CrossRef Medline

11. Zivadinov R, Heininen-Brown M, Shirda CV, et al. Abnormal subcortical deep-gray matter susceptibility-weighted imaging filtered phase measurement in patients with MS: a case-control study. Neuroimage 2012;59:331-39 CrossRef Medline

12. Langkammer C, Liu T, Khalil M, et al. Quantitaive susceptibility mapping in multiple sclerosis. Radiology 2013;267:551-59 CrossRef Medline

13. Langkammer C, Schweser F, Krebs B, et al. Quantitative susceptibility mapping (QSM) as a means to measure brain iron? A post mortem validation study. Neuroimage 2012;62:1593-99 CrossRef Medline

14. Karachi C, François C, Parain K, et al. Three-dimensional cartography of functional territories in the human striatopallidal complex by using calbindin immunoreactivity. J Comp Neurol 2002;450: 122-34 CrossRef Medline

15. Lehéricy S, Ducros M, Van de Moortele PF, et al. Diffusion tensor fiber tracking shows distinct corticostriatal circuits in humans. Ann Neurol 2004;55:522-29 CrossRef Medline

16. Middleton FA, Strick PL. Basal ganglia output and cognition: evi- 
dence from anatomical, behavioral, and clinical studies. Brain Cogn 2000:42:183-20 CrossRef Medline

17. Jenkins M. Fast automated, N-dimensional phase-unwrapping algorithm. Magn Reson Med 2003:49:193-97 CrossRef Medline

18. Liu T, Khalidov I, de Rochefort L, et al. A novel background field removal method for MRI using projection onto dipole fields (PDF). NMR Biomed 2011;24:1129-36 CrossRef Medline

19. Yang GK. Computing Magnetic Susceptibility Maps from Gradient Recalled Echo MRI for use in Multiple Sclerosis Studies [thesis]. Columbus: Ohio State University; 2013

20. Rao SM; Cognitive Function Study Group of the National Multiple Sclerosis Society. A Manual for the Brief Repeatable Battery of Neuropsychological Tests in Multiple Sclerosis. New York: National Multiple Sclerosis Society; 1990

21. Janssen A, Boster A, Lee $\mathrm{H}$, et al. The effects of video-game training on broad cognitive transfer in multiple sclerosis: a pilot randomized controlled trial. J Clin Exp Neuropsychol 2015;37:285-302 CrossRef Medline

22. Li W, Wu B, Batrachenko A, et al. Differential developmental trajectories of magnetic susceptibility in human brain gray and white matter over the lifespan. Hum Brain Mapp 2014;35:2698-713 CrossRef Medline

23. Rudko DA, Solovey I, Gati, JS, et al. Multiple sclerosis: improved identification of disease-relevant changes in gray and white matter by using susceptibility-based MR imaging. Radiology 2014: 272: 851-64 CrossRef Medline
24. Harder SL, Hopp KM, Ward H, et al. Mineralization of the deep gray matter with age: a retrospective review with susceptibilityweighted MR imaging. AJNR Am J Neuroradiol 2008;29:176-83 CrossRef Medline

25. Penke L, Valdés Hernandéz MC, Maniega SM, et al. Brain iron deposits are associated with general cognitive ability and cognitive aging. Neurobiol Aging 2012;33:510-17.e2 CrossRef Medline

26. Glatz A, Valdés Hernández MC, Kiker AJ, et al. Characterization of multi-focal T2*-weighted MRI hypointensities in the basal ganglia of elderly community-dwelling subjects. Neuroimage 2013;82: 470-80 CrossRef Medline

27. Nigg JT. On inhibition/disinhibition in developmental psychopathology: views from cognitive and personality psychology and a working inhibition taxonomy. Psychol Bull 2000;126:220-46 CrossRef Medline

28. Brydges CR, Anderson M, Reid CL, et al. Maturation of cognitive control: delineating response inhibition and interference suppression. PLoS One 2013;8:e69826 CrossRef Medline

29. Bunge SA, Dudukovic NM, Thomason ME, et al. Immature frontal lobe contributions to cognitive control in children: evidence from fMRI. Neuron 2002;33:301-11 CrossRef Medline

30. Feekes JA, Cassell MD. The vascular supply of the functional compartments of the human striatum. Brain 2006:129:2189-201 CrossRef Medline 